\title{
Discovery of Two Cyanobacterial Phenylalanine Ammonia Lyases: Kinetic and Structural Characterizationt,‡
}

\author{
Michelle C. Moffitt $§, ¥$, Gordon V. Louie $\llbracket$, Marianne E. Bowman I, Janelle Pence ${ }^{\ddagger}$, Joseph P. \\ Noel ${ }^{\star}, \boldsymbol{I}$, and Bradley S. Moore ${ }^{\star}, \S, ¥$ \\ §Scripps Institution of Oceanography and Skaggs School of Pharmacy and Pharmaceutical Sciences, \\ University of California San Diego, La Jolla, CA, 92093 \\ $¥$ College of Pharmacy, University of Arizona, Tucson, AZ, 85721 \\ THoward Hughes Medical Institute, Jack H. Skirball Center for Chemical Biology and Proteomics, The Salk \\ Institute for Biological Studies, La Jolla, CA 92037
}

\begin{abstract}
Phenylalanine ammonia lyase (PAL)1 catalyzes the deamination of phenylalanine to cinnamate and ammonia. While PALs are common in terrestrial plants where they catalyze the first committed step in the formation of phenylpropanoids, only a few prokaryotic PALs have been identified to date. Here we describe for the first time PALs from cyanobacteria, in particular Anabaena variabilis ATCC 29413 and Nostoc punctiforme ATCC 29133, identified by screening the genome sequences of these organisms for members of the aromatic amino acid ammonia lyase family. Both PAL genes associate with secondary metabolite biosynthetic gene clusters as observed for other eubacterial PAL genes. In comparison to eukaryotic homologues, the cyanobacterial PALs are $20 \%$ smaller in size, but share similar substrate selectivity and kinetic activity toward L-phenylalanine over L-tyrosine. Structure elucidation by protein x-ray crystallography confirmed that the two cyanobacterial PALs are similar in tertiary and quatenary structure to plant and yeast PALs as well as the mechanistically related histidine ammonia-lyases.
\end{abstract}

Phenylalanine ammonia lyases (PAL, EC 4.3.1.5) catalyze the formation of trans-cinnamate via the non-oxidative deamination of L-Phe (1). PALs are ubiquitous in plants and catalyze the first committed step in the biosynthesis of several classes of plant phenylpropanoids. PALs are also commonly found in fungi. However only a few examples of prokaryotic PALs are known and these are associated with the biosynthesis of the secondary metabolites enterocin, cinnamide and 3,5-dihydroxy-4-isopropylstilbene in the microorganisms Streptomyces maritimus, Streptomyces verticillatus and Photorhabdus luminescens, respectively (2-4). PALs are members of a superfamily of ammonia lyases that also includes histidine ammonia lyase (HAL, EC 4.3.1.3) and tyrosine ammonia lyase (TAL). HALs, which are common in both eukaryotes and prokaryotes, share a common core three-dimensional structure with PALs

\footnotetext{
${ }^{\dagger}$ Research was supported by the National Institutes of Health (A147808) to B.S.M. and the National Science Foundation (Grant No. 0236027) to J.P.N.

\$Atomic coordinates and structure factors have been deposited in the Protein Data Bank, www.pdb.org [PDB ID codes ??? (AvPAL) and ??? (NpPAL)].

${ }_{1}^{1}$ Abbreviations: PAL, phenylalanine ammonia lyase; AvPAL, Anabaena variabilis phenylalanine ammonia lyase; NpPAL, Nostoc punctiforme phenylalanine ammonia lyase; HAL, histidine ammonia lyase; MIO, 4-methylidene-imidazole-5-one; PKU, phenylketonuria; rmsd, root mean square deviation; TAL, tyrosine ammonia lyase.

*To whom correspondence should be addressed. B.S.M.: telephone, +1-858-822-6650; fax, +1-858-558-3702; e-mail, bsmoore@ucsd.edu. J.P.N.: telephone, +1-858-453-4100 x1442; fax, +1-858-597-0855; e-mail, noel@ salk.edu.
} 
and catalyze the deamination of L-His in the histidine degradation pathway (1). A few TALs that specifically deaminate L-Tyr to $p$-coumarate have been isolated to date; $p$-coumarate is used as a chromophore in the photoactive yellow protein of several species of purple phototropic bacteria, including Rhodobacter capsulatus, and is also utilized in the biosynthesis of the Saccharothrix natural product, saccharomicin $(5,6)$. Recently, phenylalanine and tyrosine ammonia mutases were identified and found to be members of this extended superfamily. The aminomutases evolved specialized functions for the conversion of L-Phe or L-Tyr to the corresponding $\beta$-amino acids, the latter of which are then incorporated into the antitumor natural products taxol and C-1027, respectively $(7,8)$.

Crystal structures of HAL from the prokaryote Pseudomonas putida and PALs from parsley (Petroselinum crispum) and yeast (Rhodosporidium toruloides) have recently been reported (9-11). In total, these structure determinations showed that the ammonia-lyases exist as homotetramers possessing a conserved polypeptide-chain fold. The eukaryotic PALs are $\sim 20$ $\mathrm{kDa}$ per monomer larger than the prokaryotic HALs, by virtue of a 54-residue N-terminal extension and an inserted 122-residue domain (9-11). The additional domain in the plant and fungal enzymes forms an arch over the active site and has been proposed to function as a shielding domain by restricting substrate entry and product egress (10). Alternatively, this domain may influence the conformation of an active-site lid loop and thereby affect the stability and catalytic activity of the holoenzyme $(12,13)$. Crystallographic structures also established that the electrophilic prosthetic group required for catalytic activity in this enzyme superfamily is 4-methylidene-imidazole-5-one (MIO). MIO forms autocatalytically by cyclization and dehydration of a conserved active-site Ala-Ser-Gly tripeptide segment $(11,14)$.

Of medical significance, recombinant PAL is currently being evaluated for use in humans as enzyme substitution therapy for the treatment of the inherited metabolic disease phenylketonuria (PKU) $(13,15,16)$. This disorder results from a mutation in the enzyme phenylalanine hydroxylase, thereby impairing the elimination of surplus L-Phe derived from the diet and causing severe neurotoxic effects. PAL is a viable candidate for enzyme inclusion as it efficiently reduces elevated L-Phe levels in the blood of PKU sufferers converting excess L-Phe to the harmless metabolites cinnamic acid and ammonia. Animal models have demonstrated that the yeast Rhodosporidium toruloides PAL lowers blood L-Phe levels in PKU mice $(13,16)$. Unfortunately, in vivo studies with the $R$. toruloides PAL identified problems associated with administration, immunogenicity and stability of the enzyme.

In this study, we screened the prokaryotic genomes available in the NCBI database for putative PALs with the aim of identifying novel secondary-metabolite biosynthetic pathways and new potential PKU therapeutic enzymes. Here, we report for the first time, the identification of cyanobacterial PALs in the genomes of Nostoc punctiforme PCC73102 and Anabaena variabilis ATCC29413 and the biochemical characterization of these PALs in both wild type and mutant forms. Protein x-ray crystallographic structure determination of the A. variabilis and $N$. punctiforme PALs reported here constitute the first structural descriptions of bacterial PALs and identify the major structural differences between the eukaryotic and prokaryotic PALs.

\section{MATERIALS AND METHODS}

\section{Construction of a heterologous expression vector}

Anabaena variabilis ATCC29413-U culture was obtained from the American Type Culture Collection (ATCC). N. punctiforme ATCC29133 culture was provided by Professor D. H. Sherman, University of Michigan. Genomic DNA was isolated using a sodium dodecyl sulfatelysozyme-based method as previously described (17). Genes encoding the putative PALs were amplified by PCR from A. variabilis genomic DNA using the primers AvppF.BamHI (5'- 
GGCGGATCCATGAAGACACTATCTCAAG-3') and AvppR.NotI (5'-

GTGCGGCCGCTTAATGCAAGCAGGGTAAG-3') and $N$. punctiforme genomic DNA using the primers NppF.BamHI (5'- GGCGGATCCATGAATATAACATCTCTAC-3') and NppR.HindIII (5' - CGCAAGCTTTTACGTTGACTTTAAGCTC-3') primers. The amplified genes were cloned using the Zero Blunt ${ }^{\circledR}$ PCR cloning kit (Invitrogen) as described by the manufacturer. The inserts were excised from the resulting plasmids via BamHI and NotI or HindIII restriction digests. The gene fragment was separated from the vector DNA via agarose gel electrophoresis and purified from the agarose using the QIAquick ${ }^{\circledR}$ Gel Extraction Kit (Qiagen). Purified insert was then directionally ligated into the pHIS8 vector (18) such that the putative PAL protein would be expressed from the T7 promoter with an $\mathrm{N}$-terminal octahistidyl tag and a thrombin cleavage site to remove the affinity tag. Alternatively, $N$. punctiforme genomic DNA was purchased from ATCC (29133D) and the PAL gene (ZP_00105927) was PCR-amplified with the oligonucleotides 5'-

CACTGTCATATGAATATAACATCTCTACAACAGAACAT-3' and 5'GACAGTGGCGGCCGCTCACGTTGACTTTAAGCTCGAAAAAATATG-3'. The resulting PCR product was digested with NdeI and NotI and the $1.7 \mathrm{~kb}$ fragment was ligated into pET-28a(+) (Novagen).

\section{Construction of site directed mutants}

$A v$ PAL mutants were generated using the QuikChange ${ }^{\circledR}$ II Site-Directed Mutagenesis Kit (Stratagene, La Jolla, CA). L108A and the L108G mutants were obtained using the L108A.F primer (5'- CAACTTAGTTTGGTTCGCGAAAACAGGTGCAGGG-3') or the L108G.F primer (5'-CAACTTAGTTTGGTTCGGTAAAACAGGTGCAGGG-3'), with the corresponding reverse complement oligonucleotide primer (mutations highlighted in bold). The AvPAL expression vector pMCM2571 DNA was used as a template. Mutations in the $A \nu \mathrm{PAL}$ gene were confirmed by sequencing.

\section{Expression and Purification of His-tagged PAL}

For expression of either wild type or mutant $A v \mathrm{PAL}$, a single colony of E. coli Rosetta 2(DE3) (Novagen), transformed with the pHIS8-AvPAL plasmid, was incubated in $4 \mathrm{ml}$ of LB medium containing $50 \mathrm{mg} . \mathrm{l}^{-1} \mathrm{kanamycin}$ at $37^{\circ} \mathrm{C}$ for $16 \mathrm{hrs}$. A flask containing $75 \mathrm{ml}$ of TB medium with $50 \mathrm{mg} . \mathrm{l}^{-1}$ kanamycin was inoculated with $2 \%(\mathrm{v} / \mathrm{v})$ of the overnight seed culture. The expression culture was grown at $37^{\circ} \mathrm{C}$ to an $\mathrm{OD}_{600}$ of approximately 1.0. Protein expression was induced via the addition of $0.05 \mathrm{mM} \mathrm{IPTG}$, and cell growth was maintained at $22^{\circ} \mathrm{C}$ for 16-18 hrs. The cells were then collected by centrifugation, frozen and stored at $-80^{\circ} \mathrm{C}$ until needed.

For purification, thawed cells were resuspended in $12-15 \mathrm{ml}$ of purification buffer [ $50 \mathrm{mM}$ Tris- $\mathrm{HCl}$ (pH 8.0), $300 \mathrm{mM} \mathrm{NaCl}, 10 \%$ (v/v) glycerol, $5 \mathrm{mM} \beta$-mercaptoethanol] containing $10 \mathrm{mM}$ imidazole, and lysed by sonication. The insoluble fraction was separated from soluble material via centrifugation at $15,500 \mathrm{xg}$ for $30 \mathrm{~min}$. The soluble fraction was applied to a column containing $0.5 \mathrm{ml}$ of Ni-NTA-agarose (Qiagen) and then washed 3 times with $5 \mathrm{ml}$ purification buffer containing $20 \mathrm{mM}$ imidazole. The expressed protein containing the $\mathrm{N}$-terminal octahistidyl tag was eluted from the column with $5 \times 250 \mu$ l of purification buffer containing $250 \mathrm{mM}$ imidazole. Purified protein was then exchanged into TrisHCl storage buffer $[100 \mathrm{mM}$ TrisHCl (pH 8.5), 2 mM EDTA, 2 mM DTT, 10\% (v/v) glycerol] using a PD-10 column (Pharmacia). Protein was either used immediately for enzyme assays or stored overnight at $-80^{\circ} \mathrm{C}$ prior to use. All protein samples were analyzed by denaturing PAGE using $7.5 \%$ or 10 $\%$ SDS-polyacrylamide gels (Bio-Rad) and visualized using Bio-Safe coomassie stain (BioRad). The concentration of protein was determined using the Bio-Rad Protein Assay kit (BioRad) compared to a bovine serum albumin standard curve or by measuring the absorbance at 
$280 \mathrm{~nm}$ from a calculated extinction co-efficient for $N p$ PAL $0.807 \mathrm{~g}^{-1} \cdot 1 . \mathrm{cm}^{-1}$ at $280 \mathrm{~nm}$ and for $A v \mathrm{PAL}$ is $0.727 \mathrm{~g}^{-1} .1 . \mathrm{cm}^{-1}$ at $280 \mathrm{~nm}$.

For crystallization studies, $N p$ PAL was expressed using the plasmid pHIS8-NpPAL and purification was carried out essentially as described above for $A v \mathrm{PAL}$, except for the use of an E. coli expression strain PlusE (www.exptec.com) that over-expresseses the $E$. coli chaperone proteins, GroEL and GroES. Alternatively for kinetic studies, $N p$ PAL was expressed in $E$. coli BL21(DE3) cells (Stratagene) harboring pET-28-NpPAL and pGro7 (TaKaRa) and cultured in $25 \mathrm{ml} \mathrm{LB}$ with $50 \mathrm{mg} . \mathrm{l}^{-1}$ kanamycin and $20 \mathrm{mg} . \mathrm{l}^{-1}$ chloramphenicol overnight at $37^{\circ} \mathrm{C} .20 \mathrm{ml}$ of this culture was seeded into $1 \mathrm{~L}$ of $\mathrm{LB}$ medium with kanamycin, chloramphenicol, and $0.5 \mathrm{mg} .1^{-1} \mathrm{~L}$-arabinose and grown at $37^{\circ} \mathrm{C}$. At an $\mathrm{OD}_{600}$ of 0.6 , the culture was chilled on ice. After $5 \mathrm{~min}$, the culture was induced with $0.3 \mathrm{mM}$ IPTG and grown for $16 \mathrm{hr}$ at $20^{\circ} \mathrm{C}$. Cells were harvested by centrifugation.

The harvested cells were re-suspended in $100 \mathrm{ml} 50 \mathrm{mM}$ Tris $\mathrm{HCl}$ ( $\mathrm{pH}$ 7.9), $1 \mathrm{M} \mathrm{NaCl}$ and then passed two times through a pressure homogenizer (APV) operated at 12-14,000 p.s.i. The homogenizer was washed with an additional $100 \mathrm{ml}$ of buffer, and the wash was combined with the $E$. coli lysate. The combined mixture was placed in a water bath and heat-treated for $2 \mathrm{hr}$ at $55^{\circ} \mathrm{C}$, centrifuged at $10,000 \mathrm{xg}$, and then filtered through a vacuum filter flask $(0.2 \mu \mathrm{m}$, Nalgene).

The clarified lysate was passed over a column packed with $21 \mathrm{ml}$ Ni-NTA Superflow nickel resin (Qiagen), using an Akta Explorer 100 (GE Healthcare). The column was washed with 3 column volumes of wash buffer $[25 \mathrm{mM}$ sodium phosphate ( $\mathrm{pH} 7.0), 1 \mathrm{M} \mathrm{NaCl}, 50 \mathrm{mM}$ imidazole], and the His-tagged $N p$ PAL eluted with a linear gradient over 10 column volumes of the wash buffer blended with elution buffer (wash buffer containing $0.5 \mathrm{M}$ imidazole). Active fractions were pooled for further purification.

The active eluates were diluted ten-fold with $25 \mathrm{mM}$ TrisHCl ( $\mathrm{pH}$ 7.8) and loaded onto a column packed with $7.7 \mathrm{ml}$ Macroprep High Q (BioRad) resin. His-tagged NpPAL was eluted using a ten-column volume gradient from $0 \mathrm{M}$ to $0.5 \mathrm{M} \mathrm{NaCl}$. Fractions were evaluated for purity by SDS-PAGE (Invitrogen) and pooled accordingly.

\section{Phenylalanine ammonia lyase activity assays}

For all kinetic studies, assays were performed in a final volume of $500 \mu \mathrm{l}$ using $3.75 \mu \mathrm{g}$ of $A v \mathrm{PAL}$ or $25 \mu \mathrm{g}$ of $N p \mathrm{PAL}$ in $100 \mathrm{mM}$ Tris- $\mathrm{HCl}(\mathrm{pH} 8.5$ ). Following pre-incubation of assay mixtures at $37^{\circ} \mathrm{C}$ for $5 \mathrm{~min}$, assays were initiated by the addition of L-Phe at final concentrations ranging from 10 to $200 \mu \mathrm{M}$. The assays were performed at $37^{\circ} \mathrm{C}$ and the formation of cinnamate monitored continuously by UV spectroscopy at $280 \mathrm{~nm}$ ( $\varepsilon=16890$ for trans-cinnamic acid) for $5 \min (19)$.

To determine optimal $\mathrm{pH}$, assays were performed using $15 \mu \mathrm{g}$ of protein, in $100 \mathrm{mM}$ Tris- $\mathrm{HCl}$ of $\mathrm{pH}$ ranging from 7.0 to 9.75 in a final volume of $500 \mu$. Following incubation at $37^{\circ} \mathrm{C}$ for $5 \mathrm{~min}$, assays were initiated by the addition of L-Phe to a final concentration of $200 \mu \mathrm{M}$. The assays were stopped after 15 min by adding $50 \mu \mathrm{l}$ of concentrated $\mathrm{HCl}$ and the absorbance measured at $280 \mathrm{~nm}$. Additionally, assays were performed as described above, at $30^{\circ} \mathrm{C}, 37^{\circ} \mathrm{C}$, $40^{\circ} \mathrm{C}$, and $45^{\circ} \mathrm{C}$ to determine optimal temperature. To determine activity with the substrates

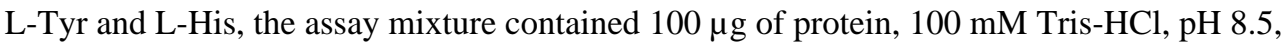
in a final volume of $1 \mathrm{ml}$. Assays were initiated by the addition of substrates to $200 \mu \mathrm{M}$ final concentration. Formation of p-coumarate and urocanate were monitored for $16 \mathrm{hr}$ at $310 \mathrm{~nm}$ and $277 \mathrm{~nm}$, respectively $(19,20)$. 


\section{Crystallization of $A$. variabilis and $\mathbf{N}$. punctiforme PALs}

Crystals of $A v \mathrm{PAL}$ and $N p \mathrm{PAL}$ were grown from 1:1 mixtures of protein solution $(\sim 11 \mathrm{mg}$ $\mathrm{ml}^{-1}$ in $12.5 \mathrm{mM}$ Tris- $\mathrm{HCl}, \mathrm{pH} 7.5,50 \mathrm{mM} \mathrm{NaCl}$ ) and a reservoir solution. The crystallization mixtures (total volume $2-3 \mu \mathrm{l}$ ) were equilibrated by hanging-drop vapor diffusion against reservoir solutions $(500 \mu \mathrm{l})$ at $4{ }^{\circ} \mathrm{C}$. The reservoir solutions contained for $A \nu \mathrm{PAL}, 0.1 \mathrm{M}$ MOPSO (pH 7.0), 7\% (w/v) polyethylene glycol 8000, $0.2 \mathrm{M}$ magnesium chloride, $10 \mathrm{mM}$ cinnamic acid, and $2 \mathrm{mM}$ dithiothreitol; and for NpPAL, $0.1 \mathrm{M}$ MOPSO (pH 7.0), 21\% (w/v) polyethylene glycol 8000, $0.2 \mathrm{M}$ lithium sulfate, and $2 \mathrm{mM}$ dithiothreitol. The AvPAL crystals grew as irregular blocks to a maximum size of $0.5 \times 0.3 \times 0.2 \mathrm{~mm}$ in the $P 1$ space-group with one homotetramer per unit cell / asymmetric unit. The $N p$ PAL crystals grew as thin needleshaped plates with maximum dimensions $0.1 \times 0.015 \times 0.01 \mathrm{~mm}$ in the $I 222$ space-group with one molecule of $N p$ PAL per asymmetric unit, in a tightly associated homotetramer generated by crystallographic symmetry.

\section{X-ray diffraction data}

For X-ray diffraction analyses, crystal samples were transferred to a cryoprotectant solution consisting of reservoir solution supplemented with $20 \%$ (v/v) glycerol, and then mounted in nylon loops for freezing by immersion in liquid nitrogen. X-ray diffraction data were measured from frozen crystals, at beamline 8.2.2 of the Advanced Light Source (Lawrence Berkeley National Laboratory) on an ADSC Quantum 315 CCD detector. Diffraction intensities were indexed, integrated, and scaled with the programs XDS and XSCALE (21), or Denzo and Scalepack (22).

\section{X-ray structure determination of $A$. variabilis and $N$. punctiforme PALs}

The initial crystallographic structure solutions were obtained through molecular-replacement analyses. Homology models were constructed with the program Modeller (23); the structure of parsley (Petroselinum crispum) PAL (PDB entry 1W27) served as a template for AvPAL, whereas a partially refined structure of $A v \mathrm{PAL}$ served as a template for $\mathrm{NpPAL}$. Monomers of the PAL homology-models were used for molecular-replacement searches with the program Molrep (24). In the initial stages of the structure determination of $A v \mathrm{PAL}$, the protein model was rebuilt extensively against an electron-density map improved by four-fold, noncrystallographic symmetry (NCS) averaging. The program Xfit (25) was used for map inspection and manipulation of the atomic model. Structural refinement used the program CNS (26). For $A v$ PAL, NCS restraints were applied until the final stages of refinement. Programs from the CCP4 (27) suite were employed for all other crystallographic calculations.

\section{RESULTS}

\section{Discovery of two cyanobacterial phenylalanine ammonia lyases}

The putative PALs from Anabaena variabilis ATCC 29413 (Ava_3988, AvPAL, 567 residues) and Nostoc punctiforme ATCC 29133 (Npun02008223, NpPAL, 569 residues) were identified by searching completed cyanobacterial genomes for homologs of the Streptomyces maritimus PAL, EncP (4). This approach identified at least two putative cyanobacterial PAL enzymes that were each annotated initially as HALs. The A. variabilis and N. punctiforme proteins were predicted to have PAL activity as they contained an aliphatic residue conserved in the active site of known PAL enzymes thought to be associated with substrate specificity (Supplementary data) (28). These two cyanobacterial PALs are 77\% identical in amino-acid sequence and have significant sequence similarity to plant PAL enzymes, although they are more similar in size ( $A \nu$ PAL is 567 residues, $N p$ PAL is 569 residues) to the smaller prokaryotic HAL enzymes ( $P$. putida HAL is 509 residues) than the larger plant PAL enzymes ( $P$. crispum PAL is 715 residues). Consistent with the association of bacterial PALs with secondary 
metabolite biosynthetic clusters, the $A v \mathrm{PAL}$ gene was identified upstream of genes encoding a mixed non-ribosomal peptide synthetase and polyketide synthase pathway, while the $N p$ PAL gene appeared to be associated with an unusual fatty acid biosynthetic gene cluster (data not shown).

\section{Expression and characterization of recombinant Anabaena and Nostoc PAL}

Gene fragments encoding the putative $A$. variabilis and $N$. punctiforme PALs were amplified by PCR from genomic DNA of these organisms and ligated into $E$. coli expression vectors. $A \nu \mathrm{PAL}$ was readily expressed as soluble His-tagged protein in $E$. coli. However, initial attempts to express the $N p$ PAL in E. coli yielded predominantly insoluble protein, and the production of significant levels of soluble His-tagged $N p$ PAL protein in E. coli required the co-expression of GroES/GroEL chaperone proteins to facilitate correct folding of the PAL protein in the host. Following Ni-NTA affinity purification of the His-tagged proteins, both cyanobacterial PALs were confirmed to be $64 \mathrm{kDa}$ in size by PAGE.

Preliminary in vitro studies confirmed that both enzymes possessed PAL activity converting L-phenylalanine to cinnamate as determined by HPLC-MS analysis with an authentic standard as a control (data not shown). Further characterization showed that $A v$ PAL had no detectable activity with L-His, although the formation of $p$-coumarate from L-Tyr could be detected after prolonged incubation. The activity of $\mathrm{Av} \mathrm{PAL}$ in Tris- $\mathrm{HCl}$ was found to be optimal at $\mathrm{pH} 8.5$ (Supplementary data) and $40^{\circ} \mathrm{C}$.

\section{Kinetic analyses of the cyanobacterial PALs}

In order to compare the activity of the two cyanobacterial PALs, kinetic studies of $A v \mathrm{PAL}$ and $N p$ PAL were conducted. In both cases, kinetic constants were estimated from linear Hanes plots. The cyanobacterial PALs were analyzed at $37^{\circ} \mathrm{C}$ and $\mathrm{pH} 8.5$ and were shown to have comparable enzymatic activity (Table 1). The $\mathrm{k}_{\text {cat }}$ of the wild type $A \nu \mathrm{PAL}$ and $N p$ PAL were determined to be approximately 500-1000 fold greater than that of the previously characterized bacterial PAL, EncP from S. maritimus $\left(\mathrm{k}_{\mathrm{cat}} 0.0048 \mathrm{~s}^{-1}, \mathrm{k}_{\mathrm{cat}} / \mathrm{K}_{\mathrm{m}} 0.21 \mathrm{mM}^{-1} . \mathrm{s}^{-1}\right.$ at $30^{\circ} \mathrm{C}, \mathrm{pH}$ 8.8) (29), but approximately 3 -fold lower than that of P. crispum PAL ( $\mathrm{k}_{\mathrm{cat}} 13.5 \mathrm{~s}^{-1}, \mathrm{k}_{\mathrm{cat}}$ / $\mathrm{K}_{\mathrm{m}} 112.5 \mathrm{mM}^{-1} . \mathrm{s}^{-1}$ at $\left.30^{\circ} \mathrm{C}, \mathrm{pH} 8.8\right)(28)$.

\section{Anabaena variabilis mutagenesis and analysis}

In previous studies of ammonia-lyases, a number of active-site residues were proposed to be responsible for conferring substrate selectivity for L-His or L-Phe $(1,28)$. One residue of interest, His 83 ( $P$. putida numbering) in HAL is thought to orient L-His in the active site, whereas the substitution of more hydrophobic residues at the corresponding position in PAL proteins may serve to better accommodate the substrate L-Phe (28). Recently, in an effort to determine the function of Val 83 in the $S$. maritimus PAL EncP, V83H and V83A mutants were generated and characterized (29). Curiously, while the EncP V83H mutant lost all lyase activity, the EncP V83A mutant was more active than the wild type enzyme with the substrate L-Phe. In this study, Ala and Gly substitutions of $A \nu \mathrm{PAL}$ were introduced at the corresponding aliphatic residue, Leu 108. Expression of both mutants in E. coli generated soluble His-tagged protein. There was a slight decrease in PAL activity of the L108A mutant as compared to the wild type enzyme (Table 1). Kinetic activity was further decreased in the L108G mutant (Table $1)$.

Based on sequence alignments, both $A v \mathrm{PAL}$ and $N p \mathrm{PAL}$ contain an additional 21 amino acid $\mathrm{N}$-terminal extension that is absent from $S$. maritimus EncP PAL and related HALs. In an effort to ascertain the role of the $\mathrm{N}$-terminal extension on $A v \mathrm{PAL}$ activity, we generated a truncated mutant by removing the first 21 amino acids. Heterologous expression of the truncated $A \nu \mathrm{PAL}$ in $E$. coli yielded soluble active protein. The kinetic parameters of the truncated mutant did not 
differ significantly from those of the wild type enzyme (Table 1). This indicates that the removal of the 21 amino acid $\mathrm{N}$-terminal region had no adverse effects on in vitro catalytic activity of $A \nu$ PAL.

\section{Crystal structure of Anabaena variabilis and Nostoc punctiforme phenylalanine ammonia lyases}

As expected from the high sequence-identity ( $75 \%$ with no insertions or deletions) between $A \nu \mathrm{PAL}$ and $\mathrm{NpPAL}$, the tertiary and quaternary structures of the two proteins (Figure $1 \mathrm{c}$, Supplementary data) are highly similar. The PALs exist as homotetramers with 222-point symmetry (Figure 1a). Within a homotetramer, each of the four identical active sites is formed by three distinct monomeric subunits. The polypeptide-chain fold of a PAL monomer is predominantly $\alpha$-helical (Figure 1a,b), with a central, up-down bundle of five $\alpha$-helices. These helices, together with a long hairpin-loop joining the last two helices of the bundle, form the inter-subunit interfaces at the core of the homotetramer. Two subdomains form at the ends of the central bundle. The N-terminal segment of the polypeptide chain constitutes the bulk of a subdomain that carries the MIO prosthetic group. The C-terminal segment forms a peripheral layer of $\alpha$-helices, which wraps around the end of the central bundle. This subdomain contributes additional intersubunit contacts that stabilize the homotetramer, and most importantly, it projects a key loop into the active-site cavity of an adjacent monomer.

The cyanobacterial PALs are very similar structurally to the PALs from parsley ( $P$. crispum; PDB entry $1 \mathrm{~W} 27$ ) and yeast (R. toruloides; 1T6P, 1Y2M) and P. putida HAL (1GKM). From a comparison of the superposed polypeptide-chain backbones of the ammonia-lyase structures, $A \nu$ PAL differs from parsley PAL by $1.4 \AA$ rmsd for 448 equivalent residues (with $36 \%$ sequence identity), from yeast PAL by $1.1 \AA$ rmsd for 463 equivalent residues ( $34 \%$ sequence identity), and from P. putida HAL by $1.5 \AA$ rmsd for 450 equivalent residues (29\% sequence identity). Interestingly, although the cyanobacterial PALs are more similar in size to the bacterial HAL (all of the bacterial ammonia lyases lack two polypeptide segments present in the eukaryotic PALs, a long N-terminal extension and an additional domain occurring near the C-terminus), the cyanobacterial PALs are more similar structurally to yeast and parsley PAL. The additional domain, termed the shielding domain (10), represents an insertion in the polypeptide chain of the eukaryotic PALs immediately after the helical bundle. In these PALs, the first $\alpha$-helix of the shielding domain extends helix -5 of the core bundle, whereas in the cyanobacterial PALs, the much shorter helix-5 is followed by a small connecting segment that leads immediately into the peripheral $\alpha$-helices of the C-terminal domain (Figure 2).

\section{MIO and modeling of substrate in the active site}

The active-site cleft of the ammonia-lyases is located above the exocyclic methylidene carbon of the MIO prosthetic group. The MIO of the cyanobacterial PALs, formed by the residues Ala167-Ser168-Gly169, is clearly defined in electron-density maps (Figure 3). The imidazolone ring is stacked against the side chain of Phe 363, with an interplanar separation of $\sim 4 \AA$. The MIO $168 \mathrm{~N}$ atom accepts a hydrogen bond from $\mathrm{Tyr}^{\mathrm{D}} 314 \mathrm{OH}$. (In this and subsequent descriptions, residue numbering refers to the polypeptide chain of a single monomer (A); residues designated with superscripted B, C, or D are contributed by a dyadrelated monomer of the homotetramer.) The other MIO atom available for polar interactions, $168 \mathrm{O}$, forms a hydrogen bond with the side chain of Asn 223, and is also directed into a small pocket lined by the backbone amide-nitrogens of Leu 171 and Gly 224 .

Although no direct structural information is available on the binding of the L-Phe substrate within the active site of the PALs, recently described crystallographic analyses of TAL from $R$. capsulatus (30) now support reliable predictive modeling. These analyses have identified in TAL an active-site His that plays a pivotal role in conferring selectivity for L-Tyr. This His 
occurs adjacent to $P$. putida-HAL position 83 , which was identified as a potential specificity determinant in earlier studies (see above). Replacement of this His by Phe effectively converts the TAL into a phenylalanine-specific ammonia lyase, and the crystal structure of this mutant TAL in complex with the reaction product cinnamic acid provides a basis for the structural modeling of L-Phe binding to native PAL enzymes. The key interactions made by the L-Phe molecule in the modeled complex with $A v \mathrm{PAL}$ (Figure 4) are a salt bridge between the substrate's $\alpha$-carboxylate and the $\delta$-guanido group of Arg 317, and an aromatic, edge-edge interaction between the substrate's phenyl ring and the side chain of Phe 107. Notably, Phe 107 replaces the key, substrate-specificity determining His in TAL. Proposed chemical mechanisms of ammonia lyase family of enzymes, based on co-crystallization of the product with TAL, are discussed by Louie et al (30).

In the cyanobacterial PAL structures, two disordered polypeptide-chain segments (residues 75-91 and 301-309) are located near the entrance to the active-site cleft. In TAL, the first of these segments is packed tightly within the active-site cleft and interacts closely with the bound substrate/product molecule, whereas the second segment forms a more external loop that caps the active-site region. These loops appear to be intrinsically flexible in the PAL proteins, as they are also poorly ordered in the structures of parsley and yeast PAL $(9,10)$. In the crystal structures of the cyanobacterial PALs, the active-site cleft appears very exposed to the external solvent, and the absence of stabilizing interactions contributed by the disordered inner loop is a likely reason for the failure to observe stable binding to these PALs of the substrate (L-Phe), the product (cinnamic acid), and a potent PAL-specific inhibitor (2-aminoindan-2-phosphonic acid) (31).

\section{DISCUSSION}

In this study, we report the discovery and characterization of phenylalanine ammonia-lyases (PAL) in the cyanobacteria Anabaena variabilis and Nostoc punctiforme. Although histidine ammonia lyases (HAL) are common in prokaryotes, PALs have been identified in the genomes of only a few prokaryotes to date. In vitro characterization confirmed that both $A v \mathrm{PAL}$ and $N p$ PAL share many characteristics with eukaryotic PALs, including an optimal $\mathrm{pH}$ of 8.5 and some relaxed selectivity possessing the ability to turnover L-Tyr. The catalytic activities of the cyanobacterial PALs were similar to those of other plant PALs, and significantly better than those of the previously characterized Streptomyces PAL, EncP (29) and Photobacteria PAL, StlA (3). Based on analysis of the ORFs flanking the PAL genes, we predict that the cyanobacterial PALs generate a cinnamic acid product that is utilized in the biosynthesis of a specialized metabolite. To the best of our knowledge, the end products of these pathways have not been identified in A. variabilis and $N$. punctiforme to date.

Elucidation of the crystal structures of A. variabilis and N. punctiforme PAL established that the cyanobacterial PALs share the same overall structural fold as previously characterized members of the aromatic amino-acid ammonia-lyase family. The eukaryotic PALs are distinguished structurally from the prokaryotic ammonia lyases by their larger size, which results primarily from the 54-residue $\mathrm{N}$-terminal extension, the inserted shielding domain and some other small inserted segments (10). Based on structural and sequence homology, the prokaryotic PALs and TALs are proposed to have evolved from bacterial HAL. Similarly, eukaryotic PAL probably evolved from bacterial HAL although this is likely to have occurred much earlier in time (10). It is therefore interesting to note that the cyanobacterial PALs, while similar in size to the bacterial HALs, are structurally more similar to the eukaryotic PALs. Thus we propose that the cyanobacterial PALs may represent an evolutionary intermediate from which the eukaryotic PALs were derived. While a number of roles for the eukaryotic specific subdomains have been proposed, studies to date of these bacterial PAL/TALs have thus far failed to elucidate a clear role for these eukaryotic-specific evolutionary additions. 
$A v \mathrm{PAL}$ and $N p \mathrm{PAL}$ both contain an $\mathrm{N}$-terminal extension of approximately 20 residues, relative to Pseudomonas putida HAL and Streptomyces maritimus EncP PAL, which is disordered in the crystal structures. The beginning of the ordered region of the polypeptide chain (Asn 25 in $A v$ PAL and Val 26 in $N p$ PAL) resides near the initial residue of the first $\beta$ strand. The N-terminal extensions of these cyanobacterial PALs therefore differ structurally from the even longer $\mathrm{N}$-terminal segment in parsley PAL, which is ordered and forms a helical segment that packs against the equivalent segment from another monomer within the homotetramer. Biochemical characterization of the truncated $A \nu \mathrm{PAL}$ with an $\mathrm{N}$-terminal deletion of 21 residues showed that enzyme activity was not significantly different from that of the full-length protein. Therefore, the $\mathrm{N}$-terminal segment is unlikely to play a role in catalysis, consistent with its positioning on the surface of the homotetramer remote from the active site. Whereas the full-length $A \nu \mathrm{PAL}$ formed crystals readily, crystallization attempts under similar conditions with the AvPAL truncated mutant were less successful, and instead yielded large amounts of aggregated protein. This observation is perhaps indicative of the altered surface properties and solubility of the truncated $A v$ PAL protein.

Mutants were generated within the predicted substrate binding pocket of $A \nu$ PAL based upon previously reported studies of wild type and V83-replaced Streptomyces PAL, EncP (29). While the Streptomyces V83A mutant enzyme showed improved catalytic activity, the corresponding Ala replacement (L108A) and also a Gly replacement (L108G) in the AvPAL enzyme resulted in decreased catalytic activity. Comparison of PAL amino-acid sequences shows the residues that form the predicted binding pocket for the L-Phe substrate's benzene ring are in general highly conserved. As expected, this binding pocket is predominantly hydrophobic in character and is formed by the side chains of Leu 104, Phe 107, Leu 108, and Leu 171 ( $A v$ PAL numbering). In $A v \mathrm{PAL}$, the slight decreases in enzyme activity caused by Ala and Gly replacements of Leu 108 are consistent with the loss of favorable nonpolar contacts to the substrate's phenyl ring. EncP PAL from S. maritimus (29) is unusual, as there are significant differences in the identity of the residues that constitute the L-Phe binding pocket (Figure 4, Supplementary data). For example, a Phe residue (403, which replaces Ile 423 in $A v \mathrm{PAL}$ ) from an adjacent monomer protrudes prominently into the substrate-binding pocket and likely provides the primary interaction surface for the phenyl ring of the L-Phe substrate. As a result, two smaller residues, Ala 82-Val 83, replace the characteristic Phe-Leu sequence. Therefore, for EncP, the altered positioning of nonpolar side chains within the substratebinding pocket may explain the atypical behavior of mutant proteins with amino-acid replacements of Val 83. An Ala substitution further enlarges the binding pocket while maintaining the pocket's nonpolar character, and results in an enhancement in enzyme activity. In contrast, replacement of Val 83 with the larger and more polar His side-chain abolishes activity.

In the future, PALs may have application in enzyme substitution therapy to reduce toxic levels of L-Phe in the blood of PKU patients by converting it to the harmless products cinnamate and ammonia $(13,15,16,32,33)$. While both the yeast and parsley PAL have been extensively evaluated as a potential therapeutic enzyme through in vivo studies, several factors have impeded their development into the clinic $(13,15,16,32,33)$. These include development of an immune response over time when administered parenterally and sensitivity to proteases when administered enterally (15). The most accessible chymotrypsin and trypsin cleavage sites within the yeast PAL are located in a flexible active-site loop $(13,15)$. While protease-protected variants of yeast PAL have been engineered, the mutant enzymes have significantly lower specific activity and a large dose of the engineered PAL is necessary to affect lowered plasma L-Phe levels. The newly discovered cyanobacterial PALs represent alternatives to the yeast PAL for future in vivo studies. 
Both cyanobacterial PALs have comparable catalytic activity to the plant and yeast enzymes and have the advantage of being significantly smaller. The smaller cyanobacterial PALs lack the protease-sensitive lid loop (13) found in all eukaryotic PALs and are predicted to be less sensitive to proteases and thus have greater in vivo stability. Other sites targeted by human proteases can be identified in the cyanobacterial PALs and engineered accordingly. We have shown in this study that the 21 aa extension at the N-terminus, which contains chymotrypsin/ trypsin target amino acids Lys, Phe and Trp, can be removed from AvPAL with no adverse effects on activity in vitro. To prevent an immune response to PAL in vivo, recent studies have shown that yeast and parsley PAL can be derivatized with PEG at surface exposed Lys residues. The chemically modified enzyme retains all catalytic activity, has an extended catalytic half life, and reduced immunogenicity when administered subcutaneously $(13,32,33)$. Similar studies could be performed with the cyanobacterial PALs. Analyses of the crystal structures show that both cyanobacterial enzymes possess eleven surface-exposed Lys residues and another three Lys residues in disordered regions are also likely to be surface exposed (Supporting information). Using these data, the pattern of PEG derivitization can be predicted. Where necessary, additional PEG attachment sites can be created by strategically engineering new surface exposed Lys residues. The biochemical and structural characterization of cyanobacterial PALs reported in this paper, together with further structure-based chemical modification studies, could be used to engineer new PKU therapeutic enzymes with beneficial structural, biochemical and/or immunogenic properties.

\section{Supplementary Material}

Refer to Web version on PubMed Central for supplementary material.

\section{ACKNOWLEDGMENT}

We are grateful to Michael Vellard, Joshua Bliesath, and Yanhong Zhang (BioMarin Pharmaceutical) for establishing the kinetic results for NpPAL and for useful discussions.

\section{REFERENCES}

1. Poppe L, Retey J. Friedel-Crafts-type mechanism for the enzymatic elimination of ammonia from histidine and phenylalanine. Angew Chem Int Ed Engl 2005;44:3668-3688. [PubMed: 15906398]

2. Bezanson GS, Desaty D, Emes AV, Vining LC. Biosynthesis of cinnamamide and detection of phenylalanine ammonia-lyase in Streptomyces verticillatus. Can J Microbiol 1970;16:147-151. [PubMed: 5437385]

3. Williams JS, Thomas M, Clarke DJ. The gene stlA encodes a phenylalanine ammonia-lyase that is involved in the production of a stilbene antibiotic in Photorhabdus luminescens TT01. Microbiology 2005;151:2543-2550. [PubMed: 16079333]

4. Xiang L, Moore BS. Inactivation, complementation, and heterologous expression of encP, a novel bacterial phenylalanine ammonia-lyase gene. J Biol Chem 2002;277:32505-32509. [PubMed: 12082112]

5. Berner M, Krug D, Bihlmaier C, Vente A, Muller R, Bechthold A. Genes and enzymes involved in caffeic acid biosynthesis in the actinomycete Saccharothrix espanaensis. J Bacteriol 2006;188:26662673. [PubMed: 16547054]

6. Kyndt JA, Meyer TE, Cusanovich MA, Van Beeumen JJ. Characterization of a bacterial tyrosine ammonia lyase, a biosynthetic enzyme for the photoactive yellow protein. FEBS Lett 2002;512:240244. [PubMed: 11852088]

7. Christenson SD, Liu W, Toney MD, Shen B. A novel 4-methylideneimidazole-5-one-containing tyrosine aminomutase in enediyne antitumor antibiotic C-1027 biosynthesis. J Am Chem Soc 2003;125:6062-6063. [PubMed: 12785829] 
8. Walker KD, Klettke K, Akiyama T, Croteau R. Cloning, heterologous expression, and characterization of a phenylalanine aminomutase involved in Taxol biosynthesis. J Biol Chem 2004;279:53947-53954. [PubMed: 15494399]

9. Calabrese JC, Jordan DB, Boodhoo A, Sariaslani S, Vannelli T. Crystal structure of phenylalanine ammonia lyase: multiple helix dipoles implicated in catalysis. Biochemistry 2004;43:11403-11416. [PubMed: 15350127]

10. Ritter H, Schulz GE. Structural basis for the entrance into the phenylpropanoid metabolism catalyzed by phenylalanine ammonia-lyase. Plant Cell 2004;16:3426-3436. [PubMed: 15548745]

11. Schwede TF, Retey J, Schulz GE. Crystal structure of histidine ammonia-lyase revealing a novel polypeptide modification as the catalytic electrophile. Biochemistry 1999;38:5355-5361. [PubMed: 10220322]

12. Pilbak S, Tomin A, Retey J, Poppe L. The essential tyrosine-containing loop conformation and the role of the C-terminal multi-helix region in eukaryotic phenylalanine ammonia-lyases. FEBS J 2006;273:1004-1019. [PubMed: 16478474]

13. Wang L, Gamez A, Sarkissian CN, Straub M, Patch MG, Han GW, Striepeke S, Fitzpatrick P, Scriver CR, Stevens RC. Structure-based chemical modification strategy for enzyme replacement treatment of phenylketonuria. Mol Genet Metab 2005;86:134-140. [PubMed: 16006165]

14. Baedeker M, Schulz GE. Autocatalytic peptide cyclization during chain folding of histidine ammonialyase. Structure 2002;10:61-67. [PubMed: 11796111]

15. Sarkissian CN, Gamez A. Phenylalanine ammonia lyase, enzyme substitution therapy for phenylketonuria, where are we now? Mol Genet Metab 2005;86:S22-S26. [PubMed: 16165390]

16. Sarkissian CN, Shao Z, Blain F, Peevers R, Su H, Heft R, Chang TM, Scriver CR. A different approach to treatment of phenylketonuria: phenylalanine degradation with recombinant phenylalanine ammonia lyase. Proc Natl Acad Sci U S A 1999;96:2339-2344. [PubMed: 10051643]

17. Neilan BA, Jacobs D, Goodman AE. Genetic diversity and phylogeny of toxic cyanobacteria determined by DNA polymorphisms within the phycocyanin locus. Appl Environ Microbiol 1995;61:3875-3883. [PubMed: 8526499]

18. Jez JM, Ferrer JL, Bowman ME, Dixon RA, Noel JP. Dissection of malonyl-coenzyme A decarboxylation from polyketide formation in the reaction mechanism of a plant polyketide synthase. Biochemistry 2000;39:890-902. [PubMed: 10653632]

19. Kalghatgi KK, Subba Rao PV. Microbial L-phenylalanine ammonia-lyase. Purification, subunit structure and kinetic properties of the enzyme from Rhizoctonia solani. Biochem J 1975;149:65-72. [PubMed: 1191266]

20. Tabor H, Mehler AH. Histidase and urocanase. Methods Enzymol 1955;2:228-233.

21. Kabsch W. Automatic processing of rotation diffraction data from crystals of initially unknown symmetry and cell constants. J Appl Crystallogr 1993;26:795-800.

22. Otwinowski Z, Minor W. Processing of X-ray diffraction data collected in oscillation mode. Methods Enzymol 1997;276:307-326.

23. Sali A, Blundell TL. Comparative protein modelling by satisfaction of spatial restraints. J Mol Biol 1993;234:779-815. [PubMed: 8254673]

24. Vagin A, Teplyakov A. MOLREP: an automated program for molecular replacement. J Appl Crystallogr 1997;30:1022-1025.

25. McRee DE. XtalView/Xfit--A versatile program for manipulating atomic coordinates and electron density. J Struct Biol 1999;125:156-165. [PubMed: 10222271]

26. Brunger AT, Adams PD, Clore GM, DeLano WL, Gros P, Grosse-Kunstleve RW, Jiang JS, Kuszewski J, Nilges M, Pannu NS, Read RJ, Rice LM, Simonson T, Warren GL. Crystallography \& NMR system: A new software suite for macromolecular structure determination. Acta Crystallogr D Biol Crystallogr 1998;54:905-921. [PubMed: 9757107]

27. Collaborative Computing Project 4 . The CCP4 suite: programs for protein crystallography. Acta Crystallogr D Biol Crystallogr 1994;50:760-763. [PubMed: 15299374]

28. Rother D, Poppe L, Morlock G, Viergutz S, Retey J. An active site homology model of phenylalanine ammonia-lyase from Petroselinum crispum. Eur J Biochem 2002;269:3065-3075. [PubMed: 12071972] 
29. Xiang L, Moore BS. Biochemical characterization of a prokaryotic phenylalanine ammonia lyase. J Bacteriol 2005;187:4286-4289. [PubMed: 15937191]

30. Louie GV, Bowman ME, Moffitt MC, Baiga TJ, Moore BS, Noel JP. Structural determinants and modulation of substrate specificity in phenylalanine - tyrosine ammonia lyases. Chem Biol. (In press)

31. Appert C, Zon J, Amrhein N. Kinetic analysis of the inhibition of phenylalanine ammonia-lyase by 2-aminoindan-2-phosphonic acid and other phenylalanine analogues. Phytochemistry 2003;62:415422. [PubMed: 12620354]

32. Gamez A, Sarkissian CN, Wang L, Kim W, Straub M, Patch MG, Chen L, Striepeke S, Fitzpatrick P, Lemontt JF, O'Neill C, Scriver CR, Stevens RC. Development of pegylated forms of recombinant Rhodosporidium toruloides phenylalanine ammonia-lyase for the treatment of classical phenylketonuria. Mol Ther 2005;11:986-989. [PubMed: 15922970]

33. Ikeda K, Schiltz E, Fujii T, Takahashi M, Mitsui K, Kodera Y, Matsushima A, Inada Y, Schulz GE, Nishimura H. Phenylalanine ammonia-lyase modified with polyethylene glycol: Potential therapeutic agent for phenylketonuria. Amino Acids 2005;29:283-287. [PubMed: 15977040]

34. Appert C, Logemann E, Hahlbrock K, Schmid J, Amrhein N. Structural and catalytic properties of the four phenylalanine ammonia-lyase isoenzymes from parsley (Petroselinum crispum Nym.). Eur J Biochem 1994;225:491-499. [PubMed: 7925471] 

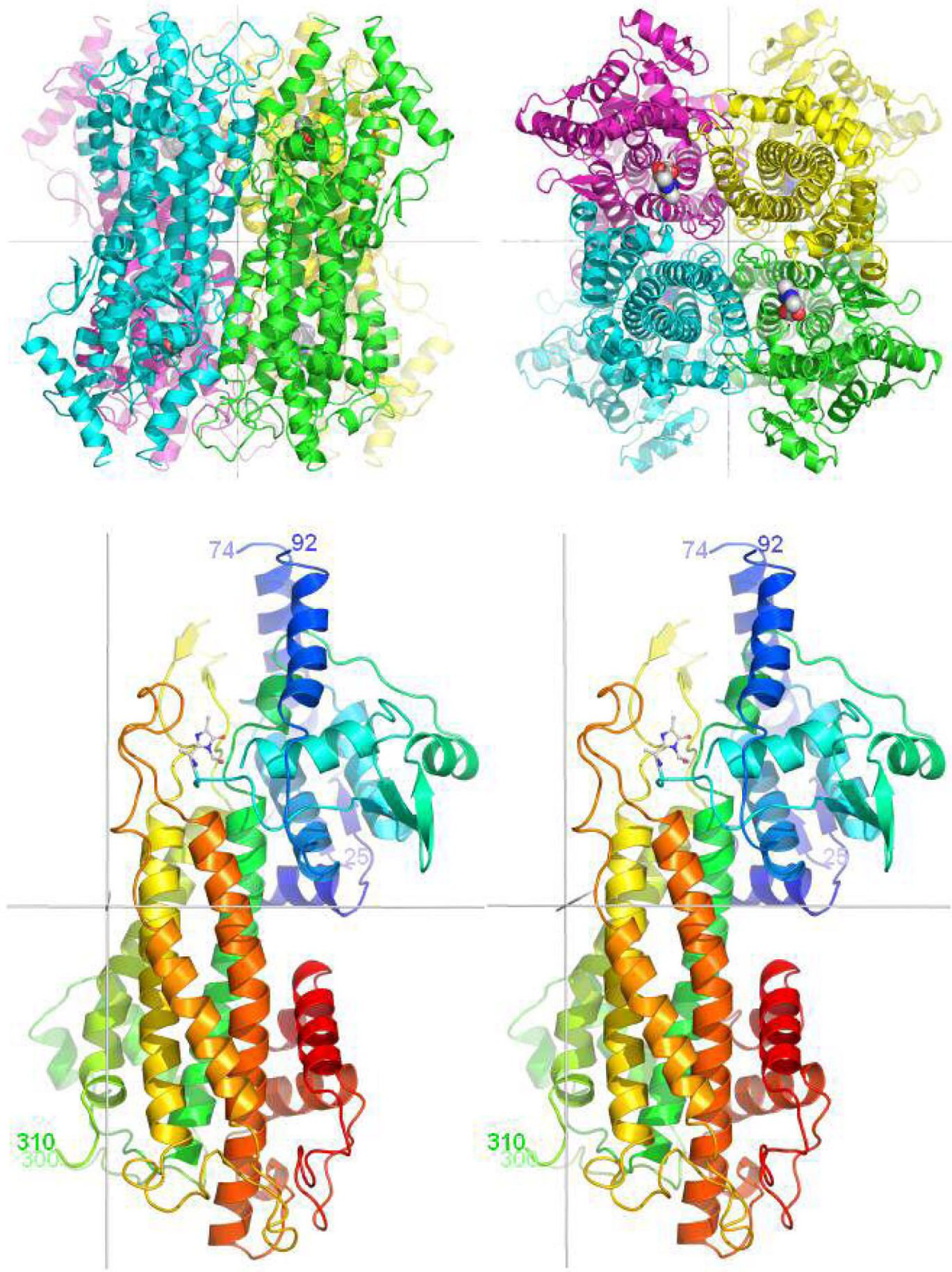

FIGURE 1. Structure of cyanobacterial PAL homotetramers and monomers

(A) Ribbon representation of the AvPAL homotetramer, with the polypeptide chains of four individual monomers A (green), B (cyan), C (magenta), and D (yellow). The atoms of the four MIO prosthetic groups are drawn as spheres. The left panel shows a view from the side of the core helical bundles of the four monomers; the right panel shows a top view, into the activesite clefts of two of the monomers. The 222 point-symmetry of the homotetramer is generated 
by three mutually orthogonal and intersecting two-fold axes (gray lines; two of the axes are visible in each orientation, with the third perpendicular to these two). (B) Stereo ribbonrepresentation of a monomer of AvPAL. The polypeptide chain is colored according to the colors of the rainbow, with blue for the $\mathrm{N}$-terminus, and red for the C-terminus. Two poorly ordered loops (residues 75 to 91 and 301 to 309) are not included in the structure. The atoms of MIO, formed by the residues Ala167-Ser168-Gly169 of this polypeptide chain are drawn as balls and sticks. The two-fold axes that relate this monomer to the other monomers in the homotetramer are shown as gray lines. This orientation of view here corresponds to a left-side view of the green monomer in Figure 1A. This orientation of view is maintained roughly in all subsequent figures, except for Figure 2. 

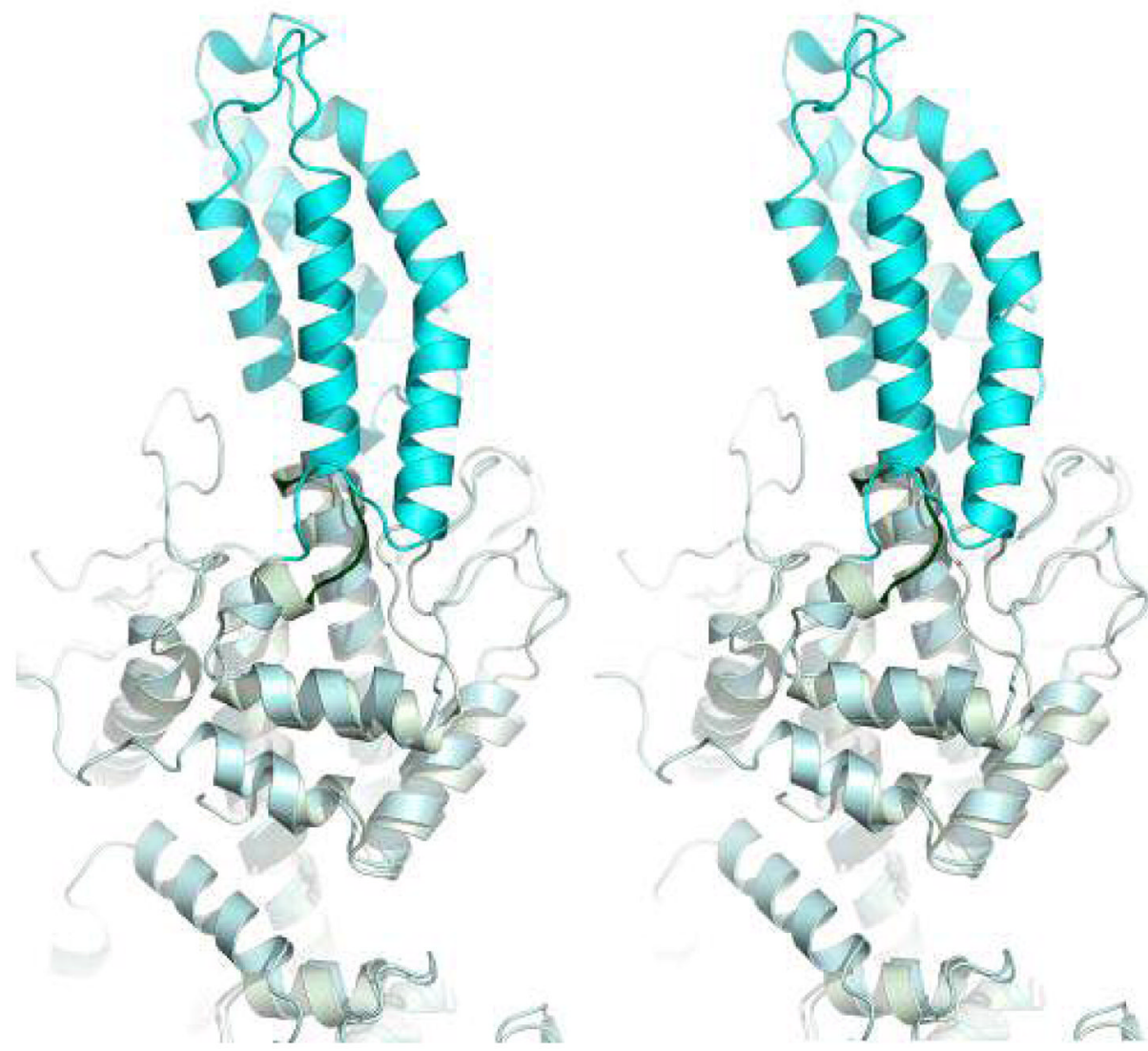

Figure 2. Comparison of the polypeptide-chain backbones of monomers of $A v P A L$ (green) and parsley PAL (cyan)

Only regions in the vicinity of the inserted shielding domain of parsley PAL (highlighted in darker cyan) are shown. In $A v \mathrm{PAL}$, the shielding domain is replaced by a short loop (dark green). 


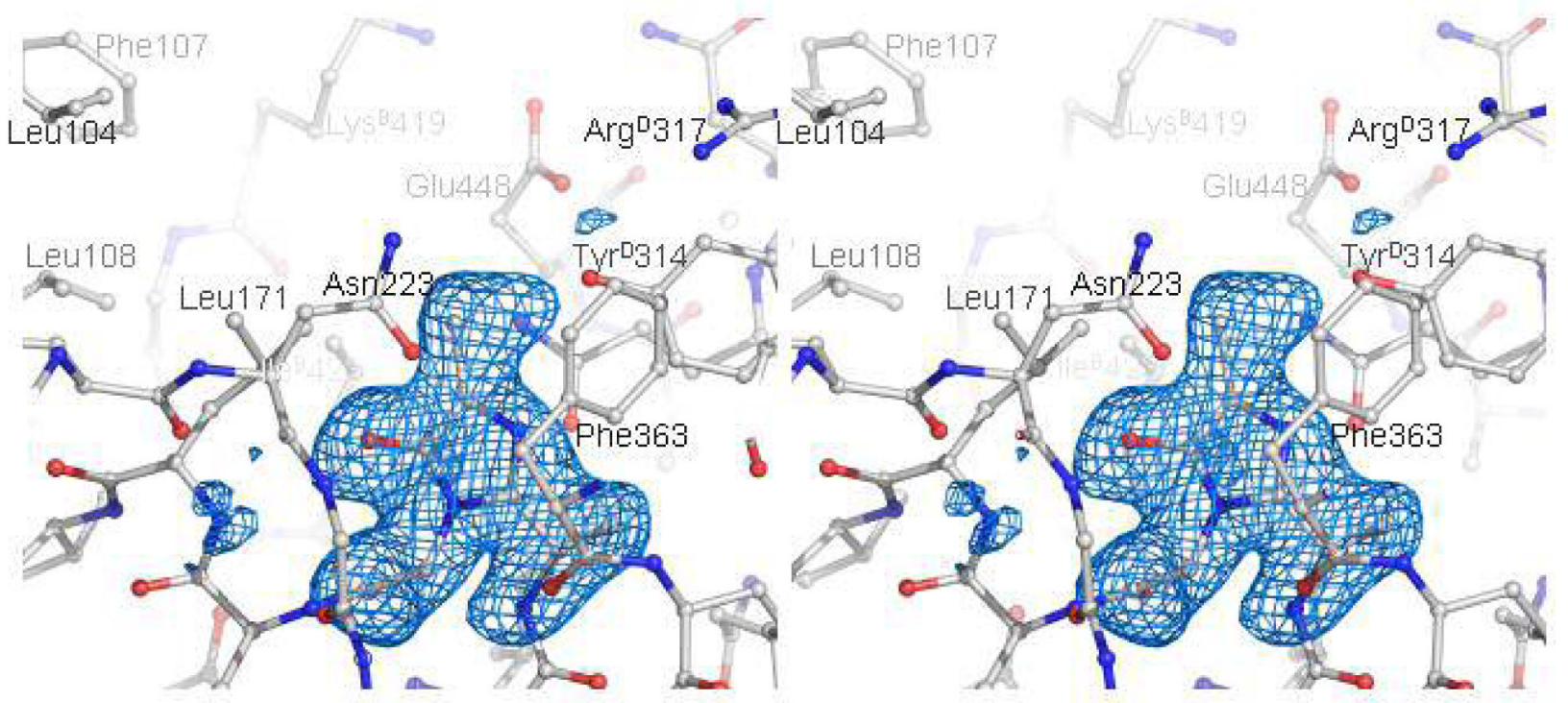

FIGURE 3. Stereo view of the methylidene-imidazolone prosthetic group and protein residues forming the active-site pocket

Protein atoms are drawn as balls and sticks, and are colored by element (carbon: grey; nitrogen: blue; oxygen: red). Hydrogen-bonding interactions formed by MIO are represented as green dashed lines. An oxyanion hole is formed by the backbone amidenitrogens of Leu 172 and Gly 225. The blue-colored contours envelope regions greater than $3 \sigma$ in the MIO-omit electrondensity map (coefficients $\left[\mathrm{F}_{\mathrm{O}}-\mathrm{F}_{\mathrm{c}}{ }^{\prime}\right], \phi_{\mathrm{c}}$ ', where $\mathrm{F}_{\mathrm{c}}$ ' and $\phi_{\mathrm{c}}$ ' were calculated without contribution from atoms of the MIO). 


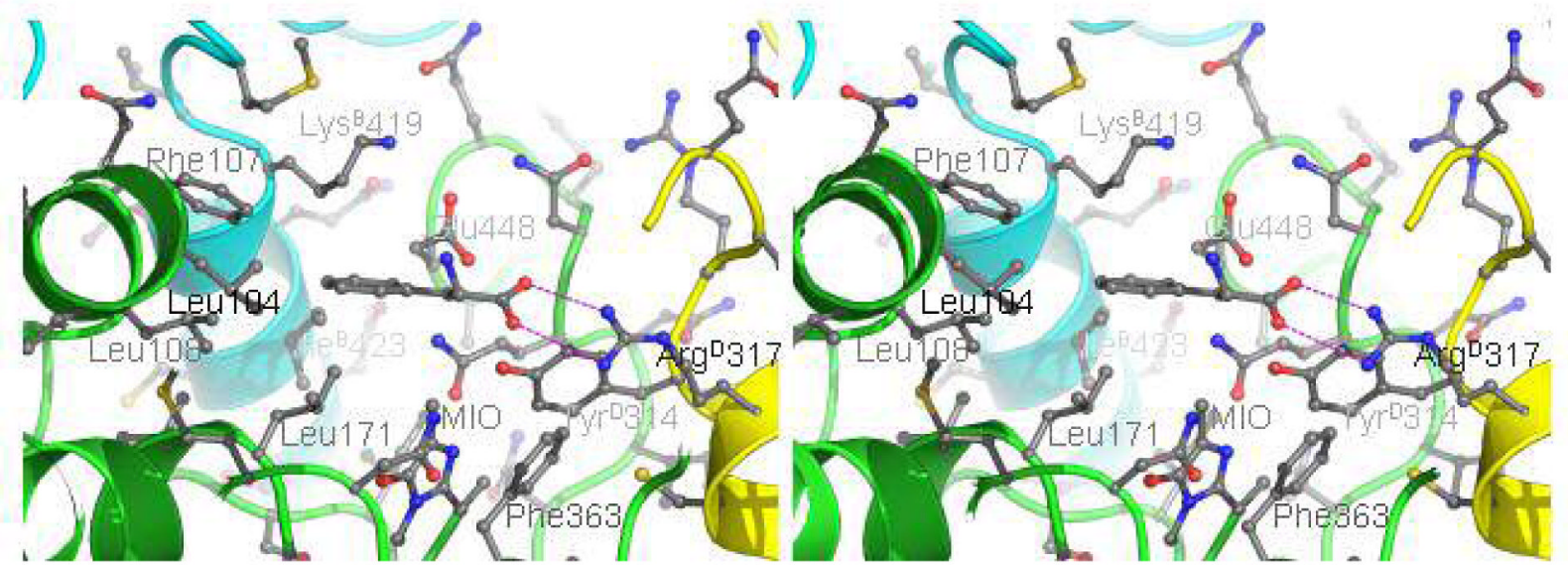

FIGURE 4. Modeled binding of the L-Phe substrate to AvPAL

Hydrogen-bonding interactions formed between the carboxylate group of the L-Phe substrate and the $\delta$-guanido group of $A \nu \mathrm{PAL}-\mathrm{Arg} 317$ are represented as magenta dashed lines.

Polypeptide-chain backbones of $A v \mathrm{PAL}$ are represented as ribbons, and colored according to monomer as in Figure 3(a). (B) Amino acid differences within the substrate-binding pockets of $A v \mathrm{PAL}$ and modeled Streptomyces maritimus are: AvPAL Phe $107=$ EncP Ala 82, Leu 108 $=$ Val 83, Lys $419=$ Met 399, Ile $423=$ Phe 403, Glu $448=$ Ala 428. 
Table 1

Kinetic analysis of $A v$ PAL and $N p$ PAL wildtype enzymes and comparison with AvPAL mutants.

\begin{tabular}{|c|c|c|c|}
\hline & $\mathbf{K}_{\mathbf{m}}(\mathbf{m M})$ & $\mathbf{k}_{\text {cat }}\left(\mathbf{s}^{-\mathbf{1}}\right)$ & $\mathrm{k}_{\text {cat }} / \mathrm{K}_{\mathrm{m}}\left(\mathrm{mM}^{-1} / \mathrm{s}^{-1}\right)$ \\
\hline *AvPAL & $0.060 \pm 0.005$ & $4.3 \pm 0.4$ & 72.2 \\
\hline${ }^{*} N p \mathrm{PAL}$ & 0.045 & 1.96 & 43.8 \\
\hline * L108A & $0.069 \pm 0.006$ & $3.6 \pm 0.3$ & 52.5 \\
\hline${ }^{*} \mathrm{~L} 108 \mathrm{G}$ & $0.07 \pm 0.02$ & $0.95 \pm 0.02$ & 14.6 \\
\hline${ }^{*} A v$ PAL $\Delta 21$ & $0.06 \pm 0.01$ & $4.6 \pm 0.8$ & 73.7 \\
\hline P. putida PAL (34) & $0.12 \pm 0.004$ & $13.5 \pm 0.1$ & 112.5 \\
\hline S. maritimus $P A L$ (29) & $0.023 \pm 0.005$ & $4.70 \times 10^{-3} \pm 0.28 \times 10^{-3}$ & 0.208 \\
\hline
\end{tabular}

* From this study. Kinetic analysis was performed at $37^{\circ} \mathrm{C}$ and $\mathrm{pH} 8.5$. 
Table 2

Statistics for X-ray diffraction data and structural refinement of $A v$ PAL and NpPAL.

\begin{tabular}{|c|c|c|}
\hline & $A v \mathbf{P A L}$ & NpPAL \\
\hline \multicolumn{3}{|l|}{ Crystal form } \\
\hline Space group & $P 1$ & $I 222$ \\
\hline Unit-cell parameters & $\begin{array}{l}\mathrm{a}=78.2, \mathrm{~b}=88.5, \mathrm{c}=90.1 \AA \\
\mathrm{a}=103.5, \mathrm{~b}=97.8, \mathrm{c}=116.2^{\circ}\end{array}$ & $\mathrm{a}=74.3, \mathrm{~b}=91.5, \mathrm{c}=151.1 \AA$ \\
\hline Monomers per asymmetric unit & 4 & 1 \\
\hline \multicolumn{3}{|l|}{$\underline{X-\text { ray diffraction data }}$} \\
\hline Resolution range $(\AA)$ & $100-1.90$ & $100-2.50$ \\
\hline Number of reflections measured & 325535 & 81381 \\
\hline Merging R-factor & $0.073(0.289)$ & $0.098(0.475)$ \\
\hline Mean I/ $\sigma \mathrm{I}$ & $7.94(3.20)$ & $12.56(1.75)$ \\
\hline Completeness & $0.948(0.884)$ & $0.929(0.697)$ \\
\hline Redundancy & $2.15(2.10)$ & $4.80(2.80)$ \\
\hline \multicolumn{3}{|l|}{ Structure refinement } \\
\hline Number of reflections used & 151571 & 15884 \\
\hline R-factor & $0.230(0.299)$ & $0.247(0.320)$ \\
\hline Free R-factor & $0.247(0.341)$ & $0.305(0.357)$ \\
\hline Number of amino-acid residues & 2056 & 513 \\
\hline Number of water molecules & 537 & 31 \\
\hline Residues with most favorable conformation (\%) & 92.2 & 92.4 \\
\hline
\end{tabular}

